\title{
Creation of database for strength calculation of constructions
}

\author{
Anatoly Bragov*, Sergey Isaev, Sergey Kapustin, Alexander Konstantinov, and Andrey Lomunov \\ Research Institute of Mechanics, National Research Lobachevsky State University of Nizhny Novgorod, 603022, Russia
}

\begin{abstract}
The problems of creation of an automated system for obtaining, processing and storing experimental information on the physical and mechanical properties of structural materials, building materials and various soil media used to provide information support for automated computing systems for analysis of strength and design of structures are discussed. The developed database is focused on studying the effects of the behavior of structural materials under highly parametric influences of force, temperature and other physical fields, as well as on equipping and experimental substantiation of mathematical models describing these effects. In the proposed variant, the database is implemented as a client-server application executed on the Microsoft.Net Framework using a powerful database management system MS SQL Server. The content of the created bank is made up of sections of primary information, material properties and material models. In order to automate the processing of data at all stages of information conversion, a special "Desktop" subsystem is included in the bank, which allows aggregating data obtained from several different experiments, correcting the selected data, processing and converting it. Processing and transformation of data in the subsystem "Desktop" can be carried out either in manual mode or on the basis of special algorithms using appropriate mathematical methods.
\end{abstract}

\section{Introduction}

The modern level of research in the field of strength of engineering structures is characterized by wide using an automated computing systems into the practice of numerical analysis of strength of engineering structures. As a rule, complex mathematical models are used to model the behavior of objects in such systems, allowing one to describe the behavior of materials used in designs in a wide range of temperature and strain rates, taking into account the influence of various media and fields of different physical nature onto the properties of these materials. In turn, the possibility of using these models is associated with the existence of a large number of material functions, for obtaining which it is necessary to conduct special "basic" experiments that allow one to identify experimentally certain effects of the behavior of the material.

The difficulty in obtaining such functions is due to the complexity of conducting "basic" experiments, as well as the need for the subsequent solution of a number of interrelated tasks:

- collection and examination of primary experimental data;

- centralized processing of primary experimental information;

- obtaining on the basis of the processed primary experimental information a material functions for a wide class of models of deformation and destruction of materials;
- checking the adequacy of the received material functions and models used in describing the actual processes in specific materials;

- accumulation, storage and examination of data at all stages of information processing;

- rapid access to data by users of various levels, as well as equipping software solutions of strength by the material models and the necessary material functions.

Therefore, the problem of creating tools that automate the collection, accumulation, mathematical processing and bringing the results of experimental research to the level of their direct use in software analysis of strength and design of structures, is currently very relevant.

This article is devoted to the description of the conceptual foundations for the creation of such tools within the framework of an automated data bank on the physical and mechanical properties of Structural Materials Database (SMD), as well as the development of the first stage of the bank's functional tools methodical, information and software for processing primary experimental information.

\section{Functional purpose and conceptual fundamentals}

The description of some information systems that provide the accumulation and access to data on the physico-mechanical properties of materials, as well as their application in software solutions to strength problems can be found in [1-7]. 
Unlike existing systems of a similar design $[1,2,7]$ the bank being developed is focused on studying the effects of the behavior of structural materials under highly parametric effects of force, temperature and other physical fields, as well as on equipping and experimental substantiation of mathematical models describing these effects.

The created bank is a logical development of previously created software tools [3-7]. In the proposed variant, the database is implemented as a client-server application executed on the Microsoft.Net Framework using a powerful MS SQL Server database. The information system provides for the possibility of remote multi-user access to data. To ensure the security of stored data, user authorization is required upon start-up, followed by an audit of the changes made and restriction of access to its individual modules and data sets, based on the roles and privileges specified by the system administrator.

In contrast to [3-6], the bank created some changes in the structure of the internal data representation, and implemented a new subsystem "Desktop" (Fig. 1), which allows to simplify and automate the way of processing information at all stages of its transformation.

A subsystem "Desktop" is a kind of space wherever you can import data from any sources: from experiments, from the finished material functions, from other desktops, in order to later expose these data to some processing. By processing is meant manual editing or application of mathematical methods implemented in external modules.

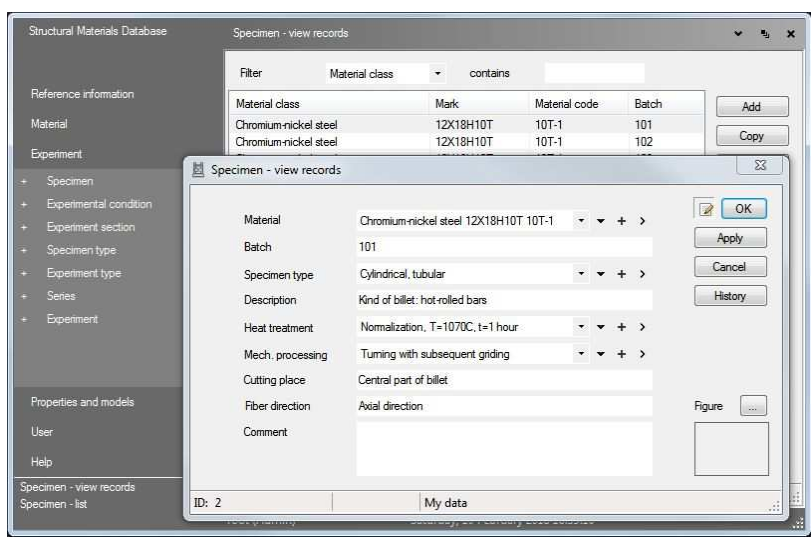

Fig. 1. Example of presentation of the subsystem "Desktop" during the input of the primary information.

The "Desktop" allows to aggregate data obtained from several different experiments, correct the selected data by processing and converting them using special algorithms and mathematical tools. The content of the created bank consists of the sections "Material", the primary information section, the material properties section, the material model section and the "Desktop" subsystem. Processing and transformation of data on the desktop can be carried out either in manual mode or on the basis of special algorithms using appropriate mathematical methods.

All listed information objects have a different degree of significance in terms of the possibility of their receipt, use and subsequent processing. In this sense, the information of the first section is the most valuable, since it is the most objective and involves the most laboriousness of obtaining it.

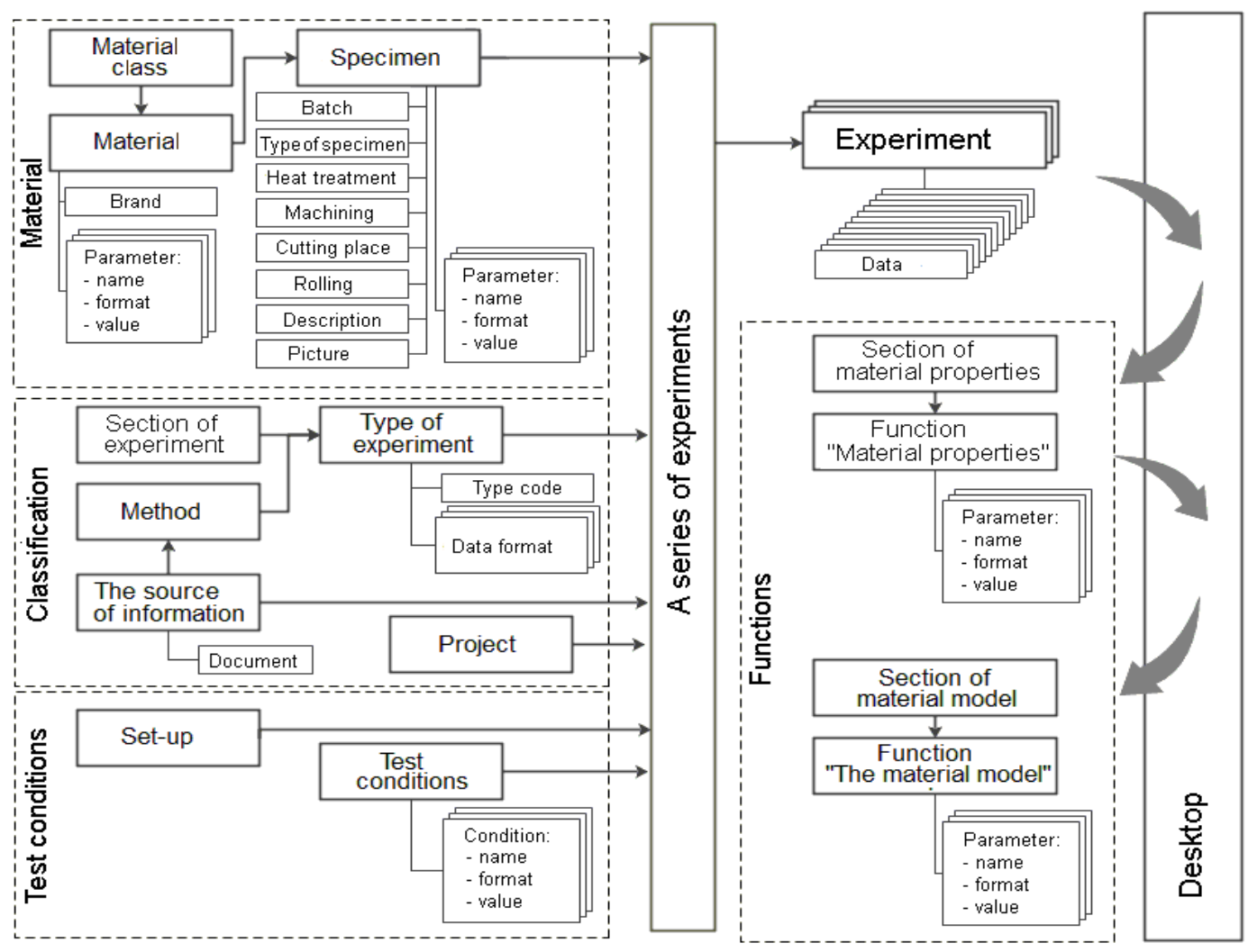

Fig. 2. Structural scheme of databank. 
The structure of the links of the basic information elements in the bank can be represented by the enlarged structural scheme shown in Fig.2.

As a result of subsequent (in particular, statistical) processing, the level of objectivity of information in the material properties section is somewhat reduced, however, it can always be restored if data of the first section are available.

The aggregate of information formed and accumulated in the bank includes a description of a wide range of objects that differ in their meaning and purpose, containing information about the material, type and condition of the experiments, the data being the direct results of the experiments (primary information), as well as data obtained as a result of the sequence of the corresponding stages transformations of primary information:

- the formation of primary functions obtained as a result of specific experiments;

- transformation and statistical processing of primary functions, as well as obtaining generalized functions of material properties on their basis;

- obtaining, on the basis of the functions of the material in the bank, the material properties of mathematical models with the help of special algorithms oriented to specific models describing the corresponding effects of the behavior of materials.

\section{Desktop functions}

All data processing begins and ends on the desktops. After the user runs the data on the desktop through a serial call of the modules it needs, a new data set (table) is obtained. And when the user has organized the desktop with the prepared data, a new function (properties or models) is created, and data is imported in it from the desktop.

The example for the 1810 stainless steel of the process of importing primary information to the desktop to prepare the "Material properties" function is shown in Fig.3.

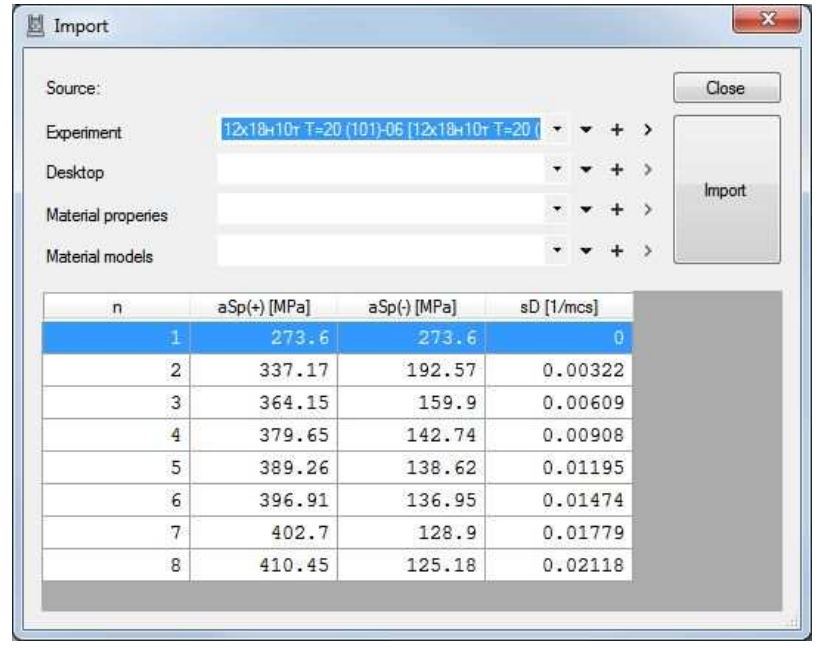

Fig. 3. The import process of primary information to the desktop from an experiment.
Importing data to the desktop is possible:

a) from the experiment (here)

b) from another desktop

c) from existing properties functions and material models.

Scheme for calling an external calculation module onto the desktop is shown in Fig.4.

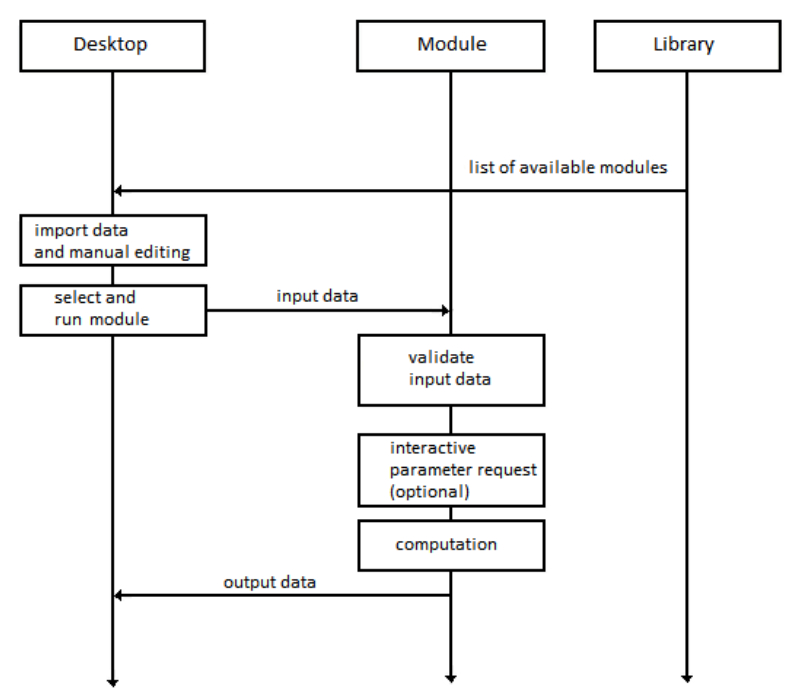

Fig. 4. Scheme for calling an external calculation module onto the desktop.

Modules called to the desktop are implemented as external (relative to the main SMD application) independent programs, which makes it possible to virtually unlimitedly scale the library of modules without rebuilding the SMD application. To exchange data between the SMD application and modules, an API (application programming interface) is developed and implemented, based on the exchange of XML packages through named pipes.

Each module has:

a. Name

b. List of output formats (data transmitted from the desktop) - optional

c. The conditions to be met by the received data

d. Interactive parameters (parameters entered by the user when the module is started) - optional

e. Mathematical algorithm for processing received data f. List of output formats - optional

The "Desktop" window with the imported data is shown in Fig.5. The color marker in the left column indicates that the data belongs to the same source (one experiment or one external source).

The module library is a separate folder on the user's disk where executable files and module configuration files should be copied. When you open the desktop, the SMD application scans the contents of this folder and collects the list of available modules.

Having prepared the data, the user launches the module having previously selected its name from the list.

When receiving data from the desktop, the module checks the applicability of this data for a number of criteria-for example, the minimum number of record groups or the number of entries in groups and so on. If the received data is correct, then in the interactive 
module will request the parameters necessary for the continuation of the operation and process the data. The result is automatically sent to the desktop, replacing the original data.

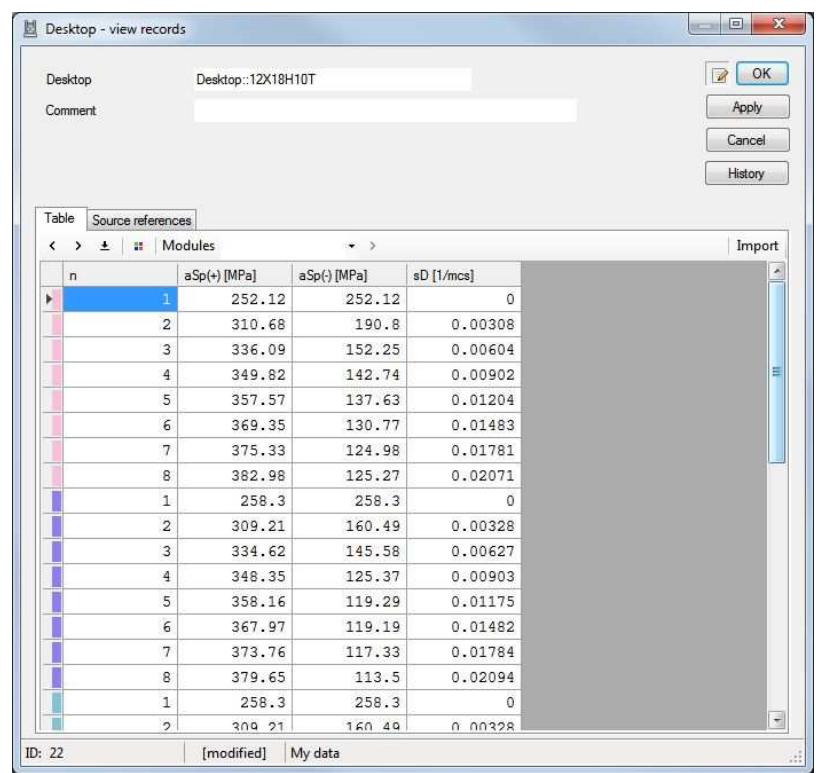

Fig. 5. The window with the imported experimental data.

External modules can be developed separately from the main SMD application in any language, it is important only that the required API is supported - this makes it possible to arbitrarily scale the library of modules and adjust it to the needs of the user.

Using the "Primary Information-Desktop-Module" scheme allows you to separate the tasks of aggregating experimental data and their mathematical processing, and the scalability and configurability of the library of external modules allows you to customize the working environment based on the specialization and needs of the researcher.

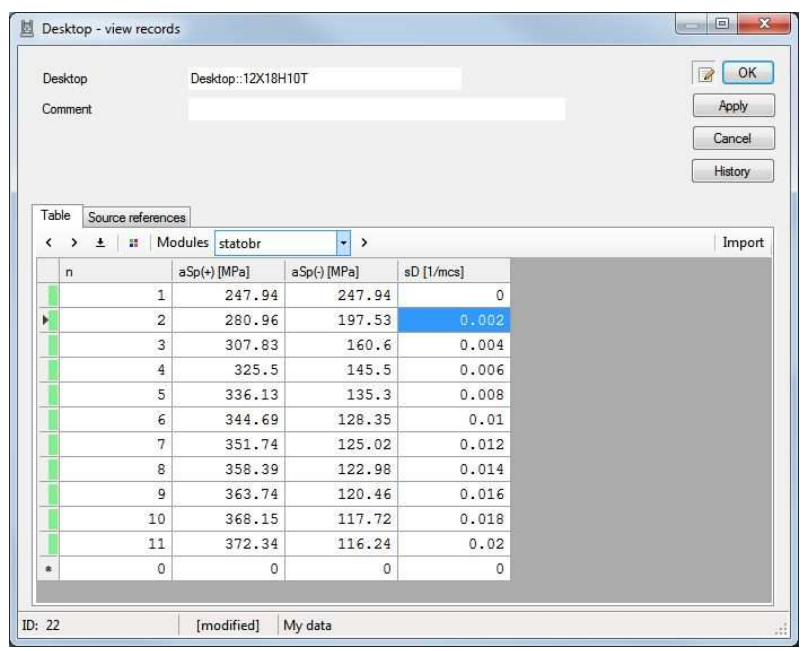

Fig. 6. The process of regularization of experimental data for the 1810 stainless steel before statistical processing.

After the input and the necessary correction of the primary information, a mutual "linking" of information is carried out for further statistical processing and approximation of the experimental information by analytic functions. The special program module analyzes the attached experimental information, identifies the presence of irregular data arrays, forms a single regular calculation grid on the required parameter (deformation, strain rate, temperature, etc.), and recalculates the original data with reference to this regular grid (Fig.6).

After the reconstruction of experimental tables with reference to a single grid, the process of their approximation by an analytic function is performed. Figure 7 shows the main window of the approximation module.

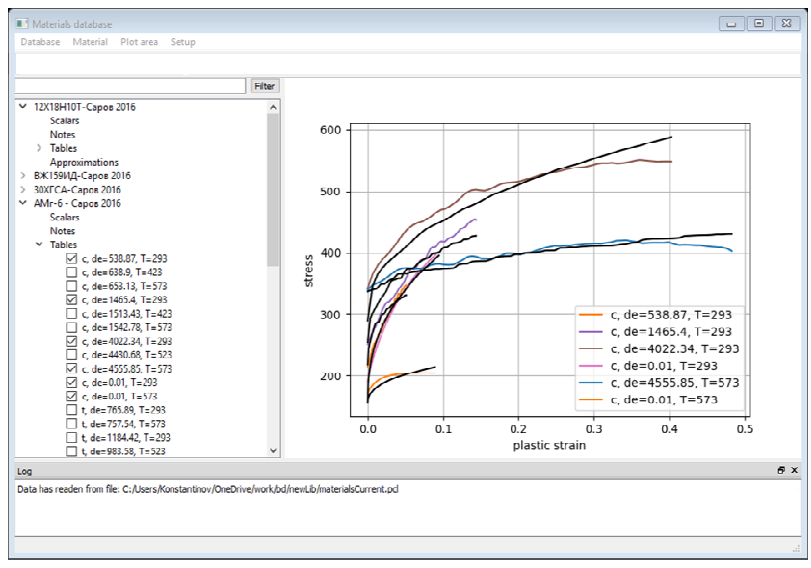

Fig. 7. The main window of approximation module.

From the left you can see the tree of experimental tables available in the database. Experiments, which are the initial data for the approximation problem, are noted. On the right, a graphical representation of the data in the form of curves in the "plastic deformation-stress" axes is shown. The colored lines correspond to the experimental curves, the black lines correspond to the diagrams calculated from the obtained analytical model.

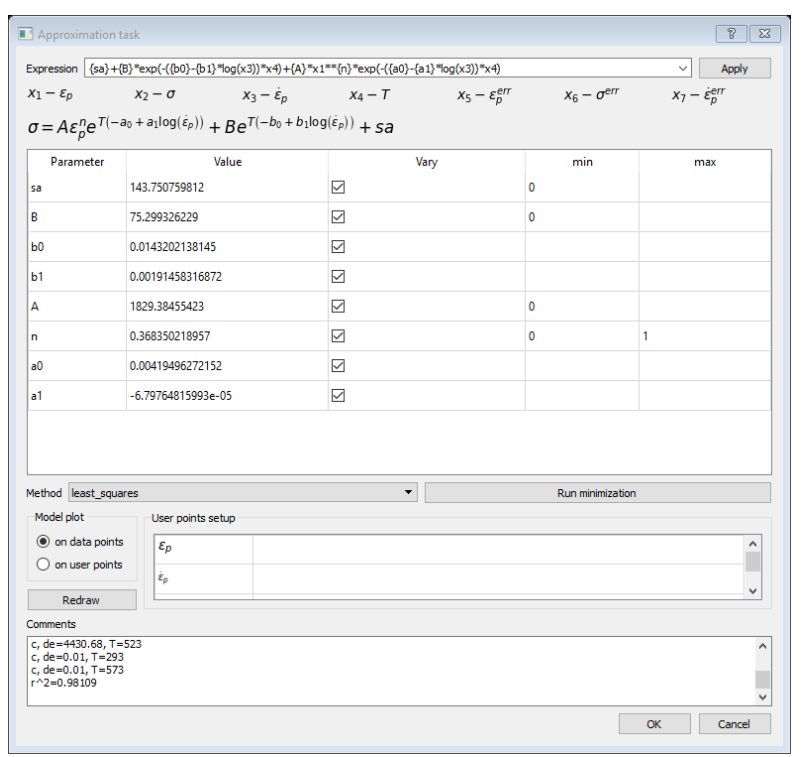

Fig. 8. The window for adjustment the parameters of the solution of the approximation problem.

Figure 8 shows the settings window for the solution of the approximation problem. Into the upper field an analytical dependence is introduced which should describe the selected experimental data. Further, this dependence is transformed into the form of a well-read mathematical formula. In the table, you can adjust the 
initial values of the model parameters, set limits on their minimum and maximum values, and also fix individual parameters. The parameterized model is stored in the database after clicking the "OK".

An important realized function of using primary information: at all stages of information processing, references to its sources are retained. Thus, as a result, the received functions of materials or material models after processing on the desktop will retain references to the experiments from which the original data was taken.

The obtained governing relationships (material models) can be used both in commercial (LS-DYNA, AUTODYN, ABAQUS, etc.) and freely distributed software products (Calculix, Impact-FEM) for modeling the behavior of structures subject to shock loads.

\section{Summary}

The structure of the automated database on the physicomechanical properties of structural materials necessary for the information support of computational systems for analysis of strength and design of critical structures has been developed. A special subsystem "Desktop" has been created that allows you to aggregate data obtained from several different experiments, correct the selected data, process and convert it.

Using MS SQL Server tools a relational model for presenting primary information was constructed including a description of the materials, experimental conditions, and numerical data of the experiments themselves.

A scheme and a set of applied software tools are implemented which allows, based on the data of numerical experiments, to generate derivative tools - the functions of material properties and the function of material models.

The developed database allows storing and using the entire data range from the initial information about the behavior of the material obtained in the experiment up to the results of validation tests used for the verification and calibration of mathematical models of material behavior.
It will be a useful tool for master-calculators of various levels of training, being both a source of ready defining relations (material models) and data necessary for the development and testing of new behavior models, numerical schemes and computational algorithms.

The work was financially supported by the Federal Targeted Program for Research and Development in Priority Areas of Development of the Russian Scientific and Technological Complex for 2014-2020 under the contract No. 14.578.21.0246 (unique identifier RFMEFI57817X0246).

\section{References}

1. Yu.D. Mishchenko, in: Ways of Development of Information Systems in Scientific Institutions: Kiev, 2 69 (1981) [in Russian]

2. V.G. Grishko, Strength of Materials 3, 115 (1981) [in Russian]

3. S.A. Kapustin, Applyied Problems of Strenght and Plasticity. Gor'kiy 17 (1984) [in Russian]

4. E.V. Bushmin, L.Ya. Dutysheva, S.A. Kapustin, Upravlyayushchie sistemy i mashiny. 3, 97 (1987) [in Russian]

4. A.M. Bragov, S.A. Kapustin, A.M. Kuznetsov, A.K. Lomunov, A.P. Bol'shakov, O.N. Ignatova, Vestnik NNGU. N. Novgorod, 4119 (2002) [in Russian]

5. S.A. Kapustin, A.M. Kuznetsov, S.O. Urlin, A.M. Bragov, A.K. Lomunov, Yu.G. Slepnev, Vestnik NNGU. N. Novgorod, 7, 141 (2006) [in Russian]

6. V.V. Belikov, N.P. Vabishchevich, Yu.V. Katyshkov, N.A. Mansurova, Matematic Modeling 26(8), 20 (2014) [in Russian]

7. A.M. Bragov, P.V. Demenko, A.K. Lomunov, J. Problems of Strength and Plasticity 64, 142 (2002) [in Russian]

8. S.A. Isaev, S.A. Kapustin, L.A. Igumnov, A.Yu. Konstantinov, A.K. Lomunov, J. Problems of Strength and Plasticity 78(3), 252 (2016) 
